\title{
A Model for the Onset of Fibrillation following Coronary Artery Occlusion
}

\author{
James P. Keener \\ Department of Mathematics, \\ University of Utah, \\ Salt Lake City, Utah 84112
}

July 10, 2003

\begin{abstract}
It is the hypothesis of this paper that the onset of fibrillation following a coronary artery occlusion is a direct consequence of the spatial inhomogeneity of chemical processes that occur following the occlusion. In particular, the localized increase of extracellular potassium and decrease of ATP availability lead to an increase of resting potential in the affected cells. This difference in potential between affected cells and normal cells drives a current, the "current of injury", which may drive oscillations in the border zone, a "border zone arrhythmia". The border zone arrhythmia may drive a "breakup instability" (related to the APD restitution instability), in the surrounding normal tissue, leading to self-sustained fibrillation.

In this paper, we present a mathematical model demonstrating this transition from normal to fibrillatory dynamics, describing the general conditions under which this transition occurs and showing in a simple ionic model the way in which spatial inhomogeneity alone can initiate self-sustained reentrant activity.
\end{abstract}

Key Words: fibrillation onset, APD alternans, ischemia, border zone arrhythmias. Acknowledgement: This research was supported in part by NSF Grant DMS-0211366. This paper is dedicated to the memory of Art Winfree.

\section{Introduction}

A coronary occlusion leading to a fatal cardiac arrhythmia is one of the leading causes of death in the industrialized world. In fact, each year in the United States, nearly a quarter of a million people die from a heart attack before reaching a hospital. Although cardiac arrhythmias and their causes have been studied for many years, there is still no adequate explanation for why or when an occlusion of a coronary artery leads to a fatal arrhythmia.

As is common knowledge, a heart attack corresponds to an occlusion of a coronary artery, leading to tissue ischemia. The affected tissue undergoes many changes including loss of oxygen, depletion of ATP, buildup of extracellular potassium, buildup of lactic acid, acidosis, increase of cell volume, failure of gap junctions, etc. $[5,6,7,45]$. It is during the 
first ten to twenty minutes following ischemia onset that a fatal arrhythmia is most likely to occur [6].

The most significant clinical arrhythmias are the reentrant arrhythmias known as ventricular tachycardia and fibrillation. Because of the inspiring theoretical work of Art Winfree [49] and the subsequent experimental verification by Ray Ideker and colleagues [8, 21], it is well known that reentrant arrhythmias can be initiated both in the laboratory and in computer models using a premature stimulus applied at a point or region that is partially refractory, leading to a graded response, with activation in one direction and failure to activate in the opposite direction. However, most clinical arrhythmias are not initiated this way. People with a coronary occlusion are not (as far as is known) subject to the external stimuli required by the Winfree paradigm. The importance of the Winfree paradigm, however, is that it emphasizes the necessity of a symmetry breaking for the formation of a reentrant pattern of excitation.

The most common explanation for the initiation of a reentrant arrhythmia is a modification of the Winfree mechanism, namely that there is a premature stimulus that arises from some point within the tissue itself which experiences a region of block as it propagates from its source and thereby initiates a reentrant pattern of activation. The one-way block could be temporary, i.e., functional, or it could be caused by various physical structures including rotational anisotropy, inhomogeneities of extracellular resistance, variations in cell-cell electrical coupling, etc. $[1,25,28,29,35]$. A related proposal is that when a normal action potential contacts a damaged region of tissue it may "break" around the obstacle and create reentrant waves in the wake $[4,40,51]$. Local conductive inhomogeneity due to spatial variations in refractoriness is often referred to as "dispersion of refractoriness" [33, 42].

While these explanations enjoy great popularity, there are numerous unresolved questions, such as "what is the mechanism of the initial premature beat and where does it originate?" [6]. It has been proposed that early after depolarizations (EAD's) or delayed after depolarizations (DAD's) [31] may provide the premature stimulus required of the Winfree paradigm to initiate reentrant patterns of electrical activity. Indeed there is a substantial body of experimental evidence supporting the arrhythmogenicity of EAD's and DAD's.

However, while each of these proposed mechanisms has merit, the connection between them and ischemia is weak. That is, it has not been demonstrated that the chemistry of ischemia onset is directly causal to any of these arrhythmogenic scenarios.

Much is known about the cellular environment during ischemia. Following the occlusion of a coronary artery, the supply of oxygen to the cell is cut off. This loss of oxygen leads quickly to a switch from aerobic to anaerobic metabolism with a resulting increased acid production (following production of lactic acid) and decreased availability of ATP. Decreased availability of ATP and increased amounts of phosphate $P_{i}$, leads to decreased Na-K ATPase activity, and subsequent increases in extracellular potassium, intracellular sodium and cell volume (due to osmotic swelling)[53]. The increase of extracellular potassium results in an increase of resting cell membrane potential, while the decrease of ATP activates $K_{A T P}$ ion channels (ATP inhibited potassium ion channels), resulting in a decreased action potential duration, by as much as $50 \%$.

At first glance, the activation of $K_{A T P}$ channels would seem to be protective. The shortening of the action potential and decrease of excitability has the important effect of decreasing the cross-bridge duty cycle, thus decreasing the need for ATP and decreasing 
the rate of cell acidification. However, the activation of $K_{A T P}$ channels is not entirely beneficial and may be proarrhythmic. It has been found that blocking $K_{A T P}$ channels with glibenclamide results in a decreased incidence of spontaneous arrhythmias while activation of these channels with bimakalim increases the incidence of spontaneous arrhythmias [43].

The classical explanation for this proarrhythmic effect is that it results from an increase in the dispersion (or spatial inhomogeneity) of the action potential duration (APD) which leads to an increased chance of conduction block leading to reentry. The dilemma with this explanation, however, is that in these experiments, application of both drugs bimakalim and glibenclamide resulted in a decrease in the observed dispersion of APD. Glibenclamide blocked $K_{A T P}$ in ischemic cells but had no effect in normal cells, while bimakalim opened $K_{A T P}$ channels in normal cells but had no amplifying effect on $K_{A T P}$ channels in ischemic tissue, in both cases rendering the APD more spatially homogeneous. Despite this homogenizing effect, the two drugs had opposite effects on the occurrence of spontaneous arrhythmias. Interestingly, bimakalim favored the occurrence of border zone spontaneous arrhythmias.

The purpose of this paper is to propose a different mechanism for the onset of reentrant arrhythmias following ischemia induced by a coronary occlusion. We illustrate this mechanism using a generic mathematical model rather than a detailed ionic model in order to show that fibrillation onset is a generic consequence of certain cellular features, not necessarily tied to only one or two specific ionic mechanisms. We also emphasize that this proposed mechanism requires a spatially distributed model, that is, spatial heterogeneity. The arrhythmogenesis that we describe here is a consequence of cellular dynamics only when cells are spatially coupled. This arrhythmogenic activity is therefore not observable in single cell models, regardless of their level of detail. On the other hand, while this model requires spatial inhomogeneity, there are no specific restrictions on the structure or geometry of the inhomogeneity. For example, there is no requirement that a narrow path with one way conduction failure be formed. In that sense, the spatial inhomogeneity is also "generic".

Our hypothesis can be stated simply: It is proposed that the chemical changes induced by ischemia, such as increased extracellular potassium and decreased availability of ATP, in a region of adequate size, are sufficient to explain the onset of fibrillation. The mechanism of this is that the increase of extracellular potassium and decrease in ATP in some region of space result in a spontaneous oscillation with short action potentials that drives an APD instability in the surrounding tissue. After a few cycles of this rhythmic behavior, portions of the expanding waves are blocked because of the nonperiodic spatial-temporal pattern of refractoriness, and reentrant waves are initiated and become self-sustained. Thus, a border zone arrhythmia drives a breakup instability, resulting directly in fibrillation, without going through the intermediate stage of a reentrant spiral pattern and without additional external stimuli.

In what follows, we show that these localized parametric changes are sufficient to initiate fibrillation. Specifically, we describe a border zone arrhythmia, the breakup instability, and how together the two are capable of inducing fibrillation. Thus, ischemia induced fibrillation onset is shown to be a generic behavior of certain excitable media with appropriate spatial inhomogeneities. Our observations are all related to a generic model of cardiac action potential dynamics of the form

$$
C_{m} \frac{\partial \phi}{\partial t}=\nabla \cdot(D \nabla \phi)-I_{i o n}(\phi, g, x)
$$


Table 1: Parameter values used for a normal cell.

\begin{tabular}{|c|l||c|c|}
\hline$\phi_{N a}$ & 1.0 & $\tau_{\text {open }}$ & $120 \mathrm{~ms}$ \\
$\phi_{K}$ & 0.01 & $\tau_{\text {close }}$ & $150 \mathrm{~ms}$ \\
$\phi_{h}$ & 0.13 & $\kappa_{h}$ & 0.02 \\
$g_{N a}$ & $3.33 \mathrm{~ms}^{-1}$ & $C_{m}$ & 1 \\
$g_{K}$ & $0.16 \mathrm{~ms}^{-1}$ & & \\
\hline
\end{tabular}

$$
\frac{d g}{d t}=G(\phi, g)
$$

where $\phi$ is the transmembrane potential, $g$ represents all local gating variables, $D$ is the spatial conductivity tensor (set to zero for isolated cells), and the $x$-dependence of the ionic current term $I_{i o n}$ reflects spatial variation in parameters due to ischemia.

The model that we use is intended to expose these generic features. For this exposition we take the ionic current to be

$$
I_{i o n}=g_{n a} m_{\infty}(\phi) h(\phi, t)\left(\phi-\phi_{N a}\right)+g_{K}\left(\phi-\phi_{K}\right),
$$

where the single dynamic gating variable $h$ is governed by

$$
\tau_{h}(\phi) \frac{d h}{d t}=h_{\infty}(\phi)-h
$$

with

$$
h_{\infty}(\phi)=f_{h}(\phi), \quad \tau_{h}(\phi)=\tau_{\text {open }}+\left(\tau_{\text {close }}-\tau_{\text {open }}\right) f_{h}(\phi), \quad m_{\infty}(\phi)=f_{m}(\phi),
$$

and $f_{h}$ and $f_{m}$ are sigmoidal functions. The model used here is a generalization of a model recently proposed by Mitchell and Schaeffer [32]. In the MS model, $f_{h}$ is a step function, and $f_{m}=F_{m}^{2}$, where $F_{m}(\phi)$ is the piecewise linear function

$$
F_{m}(\phi)=\left\{\begin{array}{cc}
0 & \phi<0 \\
\phi & 0<\phi<1 \\
1 & \phi>1
\end{array}\right.
$$

and for simplicity $\phi_{K}$ and $\phi_{N a}$ are dimensionless and scaled to 0 and 1 , respectively. Here we take $f_{h}$ to be a hyberbolic tangent $f_{h}(\phi)=\frac{1}{2}\left(1+\tanh \left(\left(\phi-\phi_{h}\right) / \kappa_{h}\right)\right.$. With $\kappa_{h}=0$ this reduces to the step function used in [32]. The parameters that we use here are listed in Table 1.

We use this model because it is simultaneously simple to understand its dynamic behavior and close enough to physical reality to mimic some important behaviors. The physiological interpretation of this model is that there is a fast inward current which is voltage gated, and a passive outward current. The inward current is gated by a fast activating variable $m$, and a time dependent inactivating variable $h$. For simplicity we refer to the inward current as a sodium current, and the outward current as a potassium current. 
While this model is simplistic, it has enough connection with reality to show the feasibility of our hypothesis. Further investigation is necessary to determine its feasibility in more detailed ionic models of ischemic or partially ischemic cells.

The two main parameters that we will manipulate in this model are the potassium Nernst potential, $\phi_{K}$ and the potassium conductance $g_{k}$. The Nernst potential changes in response to changes in extracellular potassium, and the potassium conductance changes in response to changes in $A T P$, via the $K_{A T P}$ channel.

This model has the advantage that, being a two variable model, its dynamics can be portrayed and understood using a phase portrait. The model is quite similar in structure and behavior to the reduced Hodgkin-Huxley model [27] and the Morris-Lecar model [34]. A phase portrait for this model with specified parameter values shows it to be of MorrisLecar type [34], with three rest points, a globally stable rest point, a saddle point, and an unstable node. With normal parameter values, the system is excitable, and when stimulated responds with an action potential of approximately $300 \mathrm{~ms}$ duration. The action potential duration (APD) can be calculated approximately for this model using singular perturbation arguments, since this is a "fast-slow" system.

In Fig. 1 are shown typical action potentials in response to a periodic stimulus with a basic cycle length (BCL) of $600 \mathrm{~ms}$.

\section{Border Zone Arrhythmias}

Border zones have been implicated as important regions of arrhythmogenic activity[17, 50]. A border zone is that region on the periphery of an ischemic region, with tissue and chemical properties varying nonuniformly from the ischemic region to normal tissue. The reason for spontaneous electrical activity originating in the border zone is easy to understand. A primary consequence of ischemia is that extracellular potassium rises markedly (from normal of about $4 \mathrm{mM}$ to 12 or even $20 \mathrm{mM}$ ) within the first minutes following occlusion. This increase in extracellular potassium necessarily increases the potassium Nernst potential, leading to an increase in the resting transmembrane potential of affected cells. Up to about 8 $\mathrm{mM}$ there is an increase in excitability but at higher concentrations tissue becomes inexcitable with a corresponding depolarization of resting membrane potential (about $30 \mathrm{mV}$ at 13.5 $\mathrm{mM})[12]$. As long as cells are coupled spatially, an increased resting potential in one region drives an electrotonic current, called a current of injury, into the unaffected region. This current is a depolarizing current, and if it is of sufficient amplitude can cause spontaneous oscillations in the normal tissue.

An experimental model for a border zone arrhythmia was described in [43]. In this experiment, a single cardiac fiber was placed into a two compartment bath, with one compartment providing normal conditions and the second compartment providing ischemic-like conditions. The boundary between the two zones was sharp (and therefore not physiological). In about $25 \%$ of their preparations there was spontaneous repetitive action potential activity and in about $25 \%$ there was repetitive response to extrastimili.

To understand the mechanisms behind border zone arrhythmias it is worthwhile to examine a model of two coupled cells, one normal and one with an elevated potassium Nernst 
potential. The dynamics of the two cells are governed by

$$
\begin{aligned}
& \chi_{1}\left(C_{m} \frac{d \phi_{1}}{d t}+I_{\text {ion }}^{1}\right)=\delta\left(\phi_{2}-\phi_{1}\right), \\
& \chi_{2}\left(C_{m} \frac{d \phi_{2}}{d t}+I_{\text {ion }}^{2}\right)=\delta\left(\phi_{1}-\phi_{2}\right),
\end{aligned}
$$

where $\chi_{j}$ represents the membrane surface area of the $j^{\text {th }}$ cell, which need not be identical. Let us suppose for the moment that cell 2 is the ischemic cell, having elevated rest potential and that $\frac{\delta}{\chi_{2}}$ is small. If cell 2 is rendered inexcitable by its elevated Nernst potential, then $\phi_{2}$ is fixed at its elevated rest potential. This generates a current into cell 1 that is inward if $\phi_{1}$ is below $\phi_{2}$ but outward if it is above $\phi_{2}$. If cell 1 is a normal excitable cell, this additional leak current has the ability to cause rapid oscillations, being an inward or pacemaker current when the cell is near rest but shortening action potentials by the outward current when the potential is elevated. In fact, the action potentials thus generated are always shorter than action potentials generated by a periodic depolarizing stimulus.

In Fig. 2 is shown the behavior of two coupled cells, one normal and one ischemic. Cell 1 is the excitable cell and cell 2 is inexcitable and incapable of generating an action potential $\left(\phi_{K}=0.12\right)$. When coupling is removed $(\delta=0)$ both cells approach their stable rest states. When the cells are coupled, there is a spontaneous rapid oscillation, with cell 1 having shortened action potentials and cell 2 , because it is not excitable, experiencing small oscillations that are driven by cell 1. In this simulation, the period of oscillation is about $300 \mathrm{~ms}$, with APD's of about $170 \mathrm{~ms}$. By increasing the coupling strength, we were able to find periodic oscillations with a period of $170 \mathrm{~ms}$. This is substantially faster than can be obtained with an external periodic stimulus, and is made possible by the repolarizing current from the normal cell to the ischemic cell. The normal cell cannot be driven by an external stimulus in a 1:1 rhythm at a BCL faster than about $270 \mathrm{~ms}$.

That ischemic regions can drive waves in normal tissue is demonstrated by Fig. 3. Here are shown action potentials propagating away from their source, a region of ischemic tissue. The boundary between the two regions is sharp. We emphasize that these waves occur only as a collective behavior because of spatial coupling; the dynamics of all individual cells are non-oscillatory. The period of these waves is about $325 \mathrm{~ms}$.

The boundary between normal and ischemic tissue is not sharp as it is in these simulations. In fact, a profile of extracellular potassium during ischemic conditions [11] showed highest extracellular potassium near the center of the ischemic region with a gradient of extracellular potassium as one moves toward the normal region. With such a gradient, one expects an interior region of inexcitable tissue surrounded by a region of tissue with increased excitability surrounded by the normal tissue. If the potassium gradient is not too shallow, then the electrotonic injury current can drive oscillations in the border zone of elevated excitability which in turn drives oscillations in the surrounding normal tissue. Indeed, numerical simulations on a domain with a gradual transition between the ischemic region and normal region produce waves nearly indistinguishable from those shown in Fig. 3, provided the transition is not too gradual. However, a careful quantitative study of what it means to be "too gradual" has not been done. 


\section{APD Alternans and the Breakup Instability}

It is observed in both computational models and in experiments that spiral reentrant activity is often unstable, and can lead to a breakup of waves into a constantly changing collection of small spirals, or wavelets. This "breakup instability" (also described as the multiple wavelet hypothesis) is thought to be responsible for the deterioration of monomorphic tachycardia into fibrillation, and for the maintenance of fibrillation[10].

The breakup instability is related to APD alternans. The occurrence of APD alternans in single cells is well documented. The simplest explanation for their occurrence is based on the assumption that APD is a function of the previous diastolic interval (DI). This is an approximation at best, and there is significant debate in the literature regarding the importance of other features, such as beat-to-beat memory [22, 23, 47], but it warrants attention as a first approximation.

In pacing experiments, it must be that the DI and APD add up to BCL, and since (by assumption) $A P D_{n}=A\left(D I_{n-1}\right)$,

$$
D I_{n}=B C L-A\left(D I_{n-1}\right) .
$$

If $A$ is a monotone increasing function of $D I$, then this iteration to determine $D I_{n}$ has a unique fixed point, say $D I^{*}$. This fixed point is stable if $\frac{d A\left(D I^{*}\right)}{d D I}<1$, however the fixed solution is unstable if $\frac{d A\left(D I^{*}\right)}{d D I}>1$, and the only stable solutions of (9) are periodic with period two, called APD alternans.

The model equations $(3,4)$ have this APD instability. Shown in Fig.4 is the response of the model cell to rapid pacing with $\mathrm{BCL}=270 \mathrm{~ms}$. That the equation (9) is not completely correct (since in this case the function $A(D I)$ is not monotone) is illustrated by Fig. 5 where a different response to the same stimulus protocol is seen. Here, we see a 2:1 periodic response with one full action potential for every two stimuli, with BCL $=270 \mathrm{~ms}$. Thus, this model has bistable behavior in some regions of parameter space that is not reflected by the map (9). To find the alternans, one must gradually decrease the BCL starting from a higher level (say $350 \mathrm{~ms}$ ), while the 2:1 response is found by stimulating a system that is initially at rest. (An understanding of these additional behaviors, such as Wenckebach rhythms, is provided in [15] and [23]).

The stability criterion, known as the APD restitution hypothesis, that an APD restitution curve with slope greater than one leads to APD alternans has been shown to hold in a simple model of waves on a one dimensional ring $[13,14]$, and it has been conjectured to be related to the stability of spiral waves. It is now known, however, that the APD restitution hypothesis is not precisely correct, as there are (two variable) dynamics with flat APD restitution curves whose waves on a periodic ring are unstable, and there are dynamics whose APD restitution curves are quite steep for which waves on a periodic ring are stable $[16,9]$.

In spite of the fact that the APD restitution hypothesis is not quantitatively accurate, there is still merit to the idea that an APD instability drives the spiral breakup. Indeed, simulations show that during the degradation of a single spiral, in different regions of space the APD is lengthening and shortening dynamically, and differently in different regions of space. Eventually, the APD becomes so short that it is not maintained (there is functional block), and there is a wave break, leading to the initiation of two new spiral tips, and subsequently two new spirals. This progression of events has been observed in many numerical 
studies [26, 36, 37, 38, 39] and also confirmed experimentally [41]. This breakup could also be described as resulting from dispersion of refractoriness, but because it occurs in a spatially homogeneous medium and is not a property of individual cells, it is different than what has traditionally been described as dispersion of refractoriness [33].

This same scenario can be seen with waves on a one-dimensional ring. As seen in Fig. 6, as the wave moves around the ring, it does so with constantly varying APD, until eventually the wave collapses. The important difference between one and two dimensional space is that in one dimension this collapse leads to elimination of the reentrant wave, while in two dimensions, a new double spiral core is created and the process repeats itself.

A steep restitution curve has also been shown experimentally to relate to the maintenance of spirals in that drugs that flatten the restitution curve also cause fibrillation to revert to steady spiral patterns $[46,44]$.

Two observations about the breakup instability are important. First, the instability depends solely on intrinsic dynamic properties of the medium. There are no spatial inhomogeneities required to drive this breakup so the dispersion of refractoriness is a spatio-temporal pattern induced by the dynamics of the medium, not a fixed property of individual cells. The second observation is that this breakup instability has been inappropriately associated with spirals. It is correct that the breakup creates two new spirals, but it is not the case that a spiral is needed to drive the instability. In simulations with spirals, the location of the wave break is always removed by some distance from the initial spiral core and so the wave breakup does not "know" that it is driven by a spiral. All that is needed to initiate breakup is a sufficiently rapid source of waves.

So the question that is relevant for this discussion is if it is possible to drive the breakup instability with a non-spiral source.

If the source is driven by an external stimulus, the immediate answer would appear to be no. This is because it is difficult to drive an excitable medium with external stimuli at the same high frequency as that of a spiral core.

This last observation requires clarification. The frequency of a spiral core is typically greater than $10 \mathrm{~Hz}$, and it is normally difficult to elicit action potentials in 1:1 response to stimuli that are this rapid. This was achieved for Purkinje fibers with a pace of $10 \mathrm{~Hz}$ in [20], and a high frequency focal source was achieved with local application of aconitine, a sodium channel opener [46].

However, in general, there are periodic waves that can exist at frequencies higher than those that can be stimulated by an external periodic stimulus. We already saw in the last section that our model cell can have periodic action potentials that are faster than those that can be driven by an external periodic stimulus. With a spiral wave or a border zone, the reason for this extra fast oscillation is that the APD is shortened by enhanced repolarization.

This observation provides the clue for how to stimulate high frequency waves, namely, their action potentials must be foreshortened compared to "normal" stimulated waves. Indeed, it is relatively easy to obtain high frequency response to a periodic stimulus (numerically) if the stimulus protocol includes both depolarizing and hyperpolarizing currents which significantly shorten the APD.

If there were a source of periodic waves with substantially foreshortened action potential, it might be possible to drive waves with a frequency in the range of the breakup instability. Indeed, the depletion of ATP in the ischemic region and surrounding tissue has the effect 
of shortening the action potentials of the stimulating source, thereby initiating waves of higher frequency and shorter action potential than normally possible. If these waves have a frequency similar to spiral or reentrant waves, then a breakup instability may be exposed. Of course, once a breakup instability is exposed, self-sustained reentrant waves are quickly established and within a short time, fully developed fibrillation is quickly established.

That this scenario is possible is demonstrated by numerical simulations, shown in Fig. 7 . For this figure, an ischemic zone with adjacent border zone was created by continuous variation of parameters from ischemic to normal. In the ischemic zone the tissue was inexcitable with a high resting potential $\phi_{K}=0.13$ and with elevated leak conductance $g_{k}=0.25$.

The results are as predicted. The waves that are initiated in the border zone have a higher frequency than can be maintained by the normal tissue. Thus, they propagate into the normal tissue where they meet up with the APD instability and some of the waves collapse. The dropping of an occasional action potential is of little consequence in this one dimensional simulation, however, in two spatial dimensions the consequences are significantly different. If the ischemic-border zone does not have circular symmetry so that circular symmetry is broken (which is for all practical purposes always the case), then the wave breaks occur on different waves and at different locations in space, leading to the initiation of a selfmaintained pattern of fibrillation.

Numerical simulations readily confirm this conclusion. If a localized non-circular region of elevated resting potential (simulating increased extracellular potassium) is surrounded by a narrow region with graded increased potassium conductance (simulating a border zone with decreased ATP), then in the border zone there results a spontaneous high frequency oscillation that acts as a focal source for the surrounding tissue. At first, the waves from this focal source produce a target pattern. But if the frequency of oscillation is sufficiently high, a breakup instability is exposed, leading to a pattern of self-sustained multiple reentrant waves, identified as fibrillation. A figure depicting this transition from target patterns to broken spirals is not included here, because it is nearly identical to Fig. 7 in [46]. The only difference between the two is that in our model the central region is not active, and waves originate at the border of the inactive central region, while in [46] the central region is stimulated with a periodic stimulus.

\section{Discussion}

We argue here that the initiation of self-maintained fibrillation is a generic feature of an excitable system in which there is a spatial inhomogeneity with elevated resting potential and a mechanism to shorten action potentials, here taken to be repolarization via $K_{A T P}$ channels. We demonstrate this mechanism using simulations on a simplified ionic model.

It has previously been established both numerically and experimentally [46] that a rapid focal oscillation can drive a breakup instability leading to the initiation of fibrillation. The new idea here is that the rapid focal oscillation may result naturally as a consequence of the ischemia induced changes in the local chemical environment.

This argument would be convincing were it not for the inconvenient fact that this phenomenon has not been seen in numerical simulations of more detailed ionic models such as the Beeler-Reuter [2] or Luo-Rudy I [30] models. 
There are several explanations for this failure. The first is that these standard ionic models do not exhibit APD alternans even though myocytes do. A model to reproduce APD alternans required modification of several channel dynamics [19] and similar changes were necessary to reproduce experimentally observed Wenckebach rhythms [52]. To observe the breakup instability in a two dimensional simulation, [46] found it necessary to speed up the calcium kinetics by a factor of 10 . This uncertainty about parameter values for ionic models of normal tissue is only exacerbated by the additional uncertainties about ischemic and border zone tissues.

An important determinant of arrhythmic activity is the behavior of the steady state inward current, or "window current" [30]. The window current is the steady state inward current caused by the overlap of $m_{\infty}(\phi)$ and $h_{\infty}(\phi)$,

$$
I=g_{N a} m_{\infty}(\phi) h_{\infty}(\phi)\left(\phi-\phi_{N a}\right) .
$$

In the model used here, there is a significant window current, owing to the fact that there is a range of $\phi$ for which $m_{\infty}$ and $h_{\infty}$ are simultaneously non-zero. This is also true of the Hodgkin-Huxley sodium current [24], but not of the BR sodium current. The scenario we describe here for fibrillation onset fails if there is no window current.

The window current plays little or no role in the dynamics of normal action potentials and is significant only when the resting potential of a cell is gradually elevated, as in pathological situations. The window current is important because it determines excitability as a function of rest potential. To be excitable, a resting cell must have a fast inward current for which inactivation is removed. If there is no window current, then at high resting potentials, activation of the inward current is not possible, rendering the cell inexcitable.

It is technically difficult to directly measure a sodium window current because the amplitude of the sodium current is usually quite large. However, there is indirect evidence that there should be a sodium window current in models of myocytes. This evidence comes from experimental and model studies of changes of the cellular environment during onset of ischemia. As discussed above, in the the first minutes following occlusion of a coronary artery, extracellular potassium is observed to increase. The explanation for this increase is not certain. It has been suggested that, as ATP decreases and $K_{A T P}$ channels are activated, this extra potassium current leads to the increase of extracellular potassium. However, this is now known to be incorrect as the rise in extracellular potassium is not substantially affected by glibenclamide, a specific blocker of the $K_{A T P}$ channels [48]. On the other hand, blockage of TTX-sensitive sodium channels does have the effect of reducing the increase of extracellular potassium [3], suggesting that there is a steady-state sodium current at elevated rest potentials - a window current.

Model studies corroborate these experimental observations [53]. In a model of cellular composition following an occlusion, it was found that including activation of $K_{A T P}$ channels had no effect on the rise of extracellular potassium (see also [18]). On the other hand, models with a sodium window current showed appropriate extracellular potassium efflux, while those with blocked sodium or no sodium window current, failed to do so. This leads us to believe that myocytes in an ischemic-border zone have a nontrivial sodium window current, and are therefore candidates for the generic behavior described here.

Of course, our model does not require that the window current be a sodium current, as it could also be a calcium current. In fact, in [46], alternans were found in numerical 
simulations by speeding up the kinetics of the calcium current, not the sodium current, and this was effective partly because the calcium current in the LR-I model has a significant calcium window current at high rest potentials.

\section{Conclusion}

Using general arguments and numerical simulations with generic models of excitable media, we have demonstrated that a spatial region with an elevated resting potential surrounded by a spatial region wherein action potentials are foreshortened can drive a breakup instability, leading to the rapid initiation of a fibrillatory state.

Our claim is that any mechanism that locally increases the resting potential, thereby generating a stimulating current, coupled with a mechanism that shortens the action potentials that are thereby stimulated, is capable of directly initiating fibrillation.

Since there are two well-known consequences of coronary occlusions that have these effects, namely the increase of extracellular potassium and the decrease of ATP leading to increased activity of $K_{A T P}$ channels, we suggest that these two are sufficient to explain the onset of fibrillation following a coronary occlusion.

Notice one important difference between this proposal and other proposals for the initiation of arrhythmia, namely that this proceeds directly from a border zone arrhythmia to fibrillation. There is no requirement for a reentrant circuit or reentrant spiral to be first established. As a result there is no need for any special physical structures, such as a region of one way block, or increased dispersion of refractoriness coming from the breakdown of gap junctions, etc. Of course, the breakup of circular waves into multiple spirals involves slowed conduction and localized conduction failure, but in a complicated spatial-temporal fashion that does not require any particular physical structures. This mechanism is a consequence of the collective behavior of spatially heterogeneous dynamics associated with the ischemic-border zone and is not a property of single cells.

\section{References}

[1] M. A. Allessie, F. I. M. Bonke, and F. J. G. Schopman. Circus movement in rabbit atrial muscle as a mechanism of tachycardia.II.the role of nonuniform recovery of excitability in the occurrence of unidirectional block as studied with multiple microelectrodes. Circ. Res., 39:168-177, 1976.

[2] G. W. Beeler and H. J. Reuter. Reconstruction of the action potential of ventricular myocardial fibers. J. Physiol., 268:177-210, 1977.

[3] D. M. Bers. Excitation-Contraction Coupling and Cardiac Contractile Force. Kluwer Academic Publishers, Dordrecht, the Netherlands, second edition, 2001.

[4] C. Cabo, A.M. Pertsov, J.M. Davidenko, W.T. Baxter, R.A. Gray, and J. Jalife. Vortex shedding as a precursor of turbulent electrical activity in cardiac muscle. Biophys. J., 70:1105-1111, 1996. 
[5] E. Carmeliet. Cardiac ionic currents and acute ischemia: From channels to arrhythmias. Physiological Reviews, 79(3):917-1017, 1999.

[6] W. E. Cascio. Myocardial ischemia: What factors determine arrhythmogenesis? J. Cardiovasc. Electrophys., 12:726-729, 2001.

[7] F. F. T. Ch'en, R. D. Vaughan-Jones, K. Clarke, and D. Noble. Modelling myocardial ischaemia and reperfusion. Prog. Biophys. Mol. Biol., 69:515-538, 1998.

[8] P. S. Chen, P. Wolf, E. G. Dixon, N. D. Danieley, D. W. Frazier, W. M. Smith, and R. E. Ideker. Mechanism of ventricular vulnerability to single premature stimuli in open-chest dogs. Circ. Res., 62:1191-1209, 1988.

[9] E. M. Cherry and F. H. Fenton. Suppression of alternans and conduction blocks despite steep APD restitution: Electrotonic, memory and conduction velocity restitution effects. preprint, 2003.

[10] B.-R. Choi, W. Nho, T. Liu, and G. Salama. Life span of ventricular fibrillation frequencies. Circ. Res., 91:339, 2002.

[11] R. Coronel, J. W. Fiolet, F. J. Wilms-Schopman, A. F. Schaapherder, T. A. Johnson, L. S. Gettes, and M. J. Janse. Distribution of extracellular potassium and its relation to electrophysiologic changes during acute myocardial ischemia in the isolated perfused porcine heart. Circ., 77:1125-1138, 1988.

[12] R. Coronel, F. J. G. Wilms-Schopman, L. R. C. Dekker, and M. J. Janse. Heterogeneities in $\left[\mathrm{K}^{+}\right]_{o}$ and TQ potential and the inducibility of ventricular fibrillation during acute regional ischemia in the isolated perfused porcine heart. Circ., 92:120-129, 1995.

[13] M. Courtemanche, J. P. Keener, and L. Glass. Instabilities of a propagating pulse in a ring of excitable media. Phys. Rev. Letts., 70:2182-2185, 1993.

[14] M. Courtemanche, J. P. Keener, and L. Glass. A delay equation representation of pulse circulation on a ring in excitable media. SIAM J. Appl. Math., 56:119-142, 1996.

[15] E. Cytrynbaum. Using Low Dimensional Models to Understand Cardiac Arrhythmias. PhD thesis, University of Utah, Salt Lake City, UT, 2001.

[16] E. Cytrynbaum and J. P. Keener. Stability conditions for the traveling pulse - modifying the restitution hypothesis. Chaos, 12:788-799, 2002.

[17] N. El-Sherif, R. Mehra, W. B. Gough, and R. H. Zeiler. Ventricular activation patterns of spontaneous and induced ventricular rhythms in canine one-day-old myocardial infarction: Evidence for focal and reentrant mechanisms. Circ. Res., 51:152-166, 1982.

[18] J. M. Ferrero, Jr., J. Sa‘iz, J. M. Ferrero, and N. V. Thakor. Simulation of action potential from metabolically impaired cardiac myocytes. Circ. Res., 79(2):208-221, 1996. 
[19] J. J. Fox, J. L. McHarg, and R. F. Gilmour. Ionic mechanism of electrical alternans. Am J Physiol Heart Circ Physiol, 282:H516-530, 2001.

[20] J. J. Fox, M. L. Riccio, E. Bodenschatz, and R. F. Gilmour. Spatiotemporal transition to conduction block in canine ventricle. Circ. Res., 90:289-296, 2002.

[21] D. W. Frazier, P. D. Wolf, and R. E. Ideker. Electrically induced reentry in normal myocardium - evidence of a phase singularity. PACE, 11:482, 1988.

[22] R. F. Gilmour Jr, N. F. Otani, and M. A. Watanabe. Memory and complex dynamics in cardiac Purkinje fibers. Am. J. Physiol., 97:H1826-H1832, 1997.

[23] G. M. Hall, S. Bahar, and D. J. Gauthier. Prevalence of rate-dependent behaviors in cardiac muscle. Phys. Rev. Letts., 82:2995-2998, 1999.

[24] A. L. Hodgkin and A. F. Huxley. A quantitative description of membrane current and its application to conduction and excitation in nerve. J. Physiol., 117:500-544, 1952.

[25] R. W. Joyner. Mechanisms of unidirectional block in cardiac tissues. Biophys. J., $35: 113-125,1981$.

[26] A. Karma. Spiral breakup in model equations of action potential propagation in cardiac tissue. Phys. Rev. Lett., 71:1103-1106, 1993.

[27] J. P. Keener and J. Sneyd. Mathematical Physiology. Springer-Verlag, New York, 1998.

[28] M. Lewis and P. Grindrod. One-way block in cardiac tissue: a mechanism for propagation failure in Purkinje fibres. Bull. Math. Biol., 53:881-899, 1991.

[29] T. J. Lewis and J. P. Keener. Wave-blocking in excitable media due to regions of depressed excitability. SIAM J. Appl. Math., 61:293-316, 2000.

[30] C. H. Luo and Y. Rudy. A model of the ventricular cardiac action potential; depolarization, repolarization and their interaction. Circ. Res., 68:1501-1526, 1991.

[31] C. H. Luo and Y. Rudy. A dynamic model of the cardiac ventricular action potential; II: Afterdepolarizations, triggered activity and potentiation. Circ. Res., 74:1097-1113, 1994.

[32] C. C. Mitchell and D. G. Schaeffer. A two-current model for the dynamics of cardiac membrane. preprint, 2002.

[33] G. K. Moe, W. C. Rheinbolt, and J. A. Abildskov. A computer model of atrial fibrillation. Am. Heart J., 67:200-220, 1964.

[34] C. Morris and H. Lecar. Voltage oscillations in barnacle giant muscle fiber. Biophys. J., 35:193-213, 1981.

[35] E. D. Overholt, R. W. Joyner, R. D. Veenstra, D. A. Rawling, and R. Weidmann. Unidirectional block between Purkinje and ventricular layers of papillary muscles. Am J Physiol, 247:H584-H595, 1984. 
[36] A. V. Panfilov. Spiral breakup as a model of ventricular fibrillation. Chaos, 8:57-64, 1998.

[37] A. V. Panfilov and P. Hogeweg. Spiral break-up in a modified FitzHugh-Nagumo model. Phys. Lett. A, 176:295-299, 1993.

[38] A.V. Panfilov and A.V. Holden. Self-generation of turbulent vortices in a twodimensional model of cardiac tissue. Phys.Lett.A, 147:463-466, 1990.

[39] A.V. Panfilov and A.V. Holden. Spatio-temporal irregularity in a two- dimensional model of cardiac tissue. Int.J.Bif. and Chaos, 1:119-129, 1991.

[40] A.V. Panfilov and J.P. Keener. Effects of high frequency stimulation in excitable medium with obstacle. J. Theor. Biol., 163:439-448, 1993.

[41] J. M. Pastore, S. D. Girouard, K. R. Laurita, F. G. Akar, and D. S. Rosenbaum. Mechanism linking T-wave alternans to the genesis of cardiac fibrillation. Circulation, 99:1385-1394, 1999.

[42] A. T. Peng, M. D. Lesh, W. Gibb, and A. Goel. Reentry in a model with fractal uncoupling. In Computers in Cardiology 1992. IEEE Computer Society Press, 1992.

[43] S. Picard, R. Rouet, P. Ducouret, P. Puddu, F. Flais, A. Criniti, F. Monti, and J.L. Gérard. $\mathrm{K}_{A T P}$ channels and 'border zone' arrhythmias: role of the repolarization dispersion between normal and ischaemic ventricular regions. Brit. J. Pharm., 127:1687$1695,1999$.

[44] M. L. Riccio, M. L. Koller, and R. F. Gilmour, Jr. Electrical restitution and spatiotemporal organization during ventricular fibrillation. Circ Res., 84:955-963, 1999.

[45] R. M. Shaw and Y. Rudy. Electrophysiologic effects of acute myocardial ischaemia: a theoretical study of altered cell excitability and action potential duration. Cardiovasc. Res, 35:256-272, 1997.

[46] M. Swissa, Z. Qu, T. Ohara, M. H. Lee, S. F. Lin, A. Garfinkel, H. S. Karagueuzian, J. N. Weiss, and P. S. Chen. Action potential duration restitution and ventricular fibrillation due to rapid focal excitation. J Physiol Heart Circ Physiol, 282:H1915-23, 2002 .

[47] M. A. Watanabe and M. L. Koller. Mathematical analysis of dynamics of cardiac memory and accommodation: theory and experiment. Am. J. Physiol. Heart Circ. Physiol., 282:H1534-H1547, 2002.

[48] A. A. M. Wilde, D. Escande, C. A. Schumacher, D. Thuringer, M. Mestre, J. W. T. Fiolet, and M. J. Janse. Potassium accumulation in the globally ischemic mammalian heart. a role for the atp-sensitive potassium channel. Circ. Res., 67:835-843, 1990.

[49] A. T. Winfree. Sudden cardiac death, a problem in topology. Sci. Am., 248:114-161, 1983. 
[50] A. L. Wit, S. M. Dillon, J. Coromilas, A. E. Saltman, and B. Waldecker. Anisotropic reentry epicardial border zone of myocardial infarctions. In J. Jalife, editor, Mathematical Approaches to Cardiac Arrhythmias, Ann. New York Acad. Sci. 591, pages 86-108, New York, 1990. New York Academy of Science.

[51] A. Xu and M. R. Guevara. Two forms of spiral-wave reentry in an ionic model of ischemic ventricular myocardium. Chaos, 8:157-174, 1998.

[52] A. R. Yehia, A. Shrier, K. C.-L. Lo, , and M. R. Guevara. Transient outward current contributes to Wenckebach-like rhythms in isolated rabbit ventricular cells. $A m \mathrm{~J}$. Physiol. (Heart Circ. Physiol.), 273:H1-11, 1997.

[53] C. S. Yi, A. Fogelson, J. P. Keener, and C. Peskin. A mathematical study of volume shifts and ionic concentration changes during ischemia and hypoxia. JTB, 220:83-106, 2002 . 

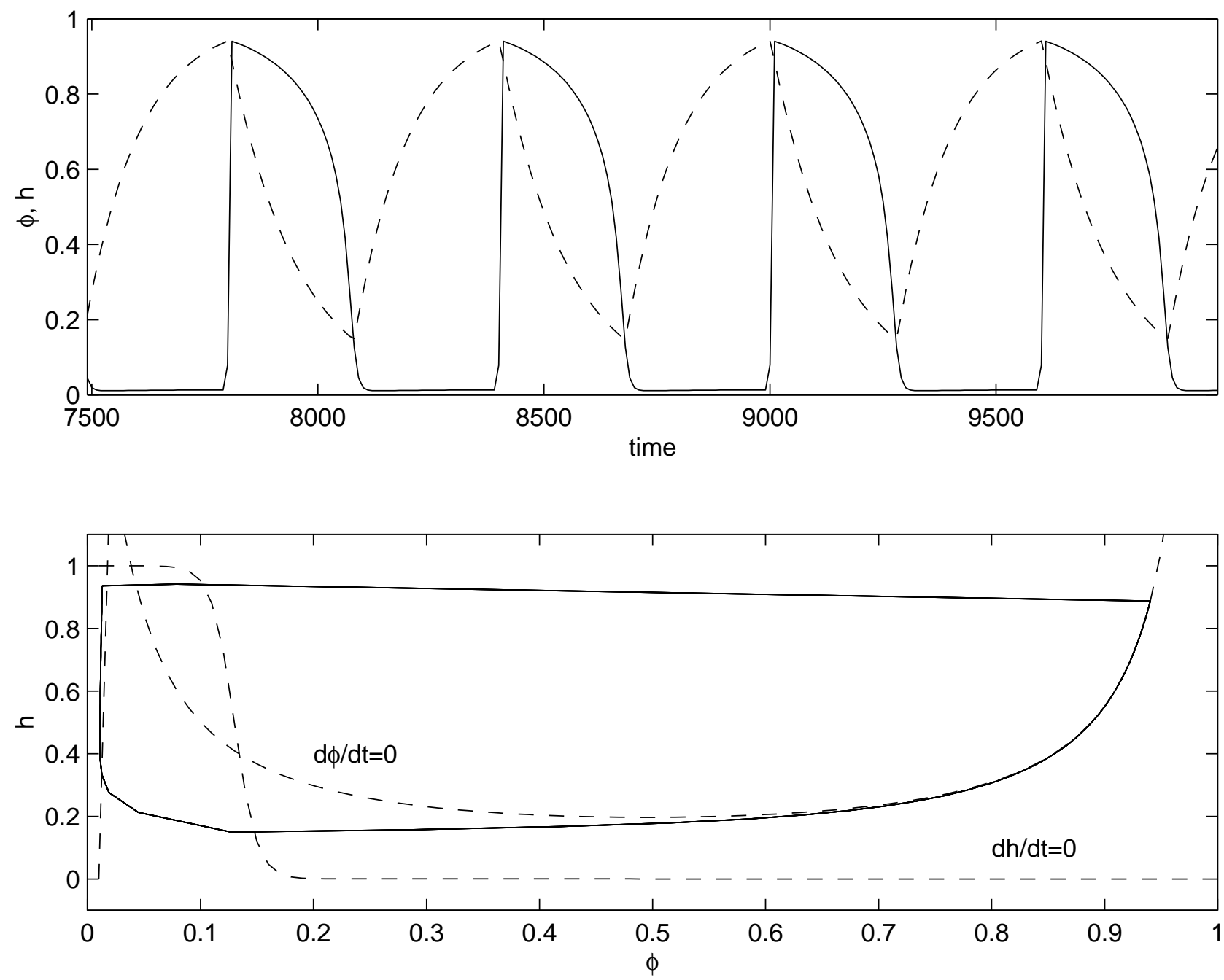

Figure 1: Action potentials of a normal cell in response to periodic stimulus with BCL of $600 \mathrm{~ms}$. The upper panel shows the potential $\phi$ and gating variable $h$ (dashed) as a function of time, while the lower panel shows the same trajectory as a phase portrait, $h$ vs. $\phi$. The dashed curves in the phase portrait are the nullclines $\frac{d \phi}{d t}=0$ and $\frac{d h}{d t}=0$ (which is $\left.h=h_{\infty}(\phi)\right)$. 

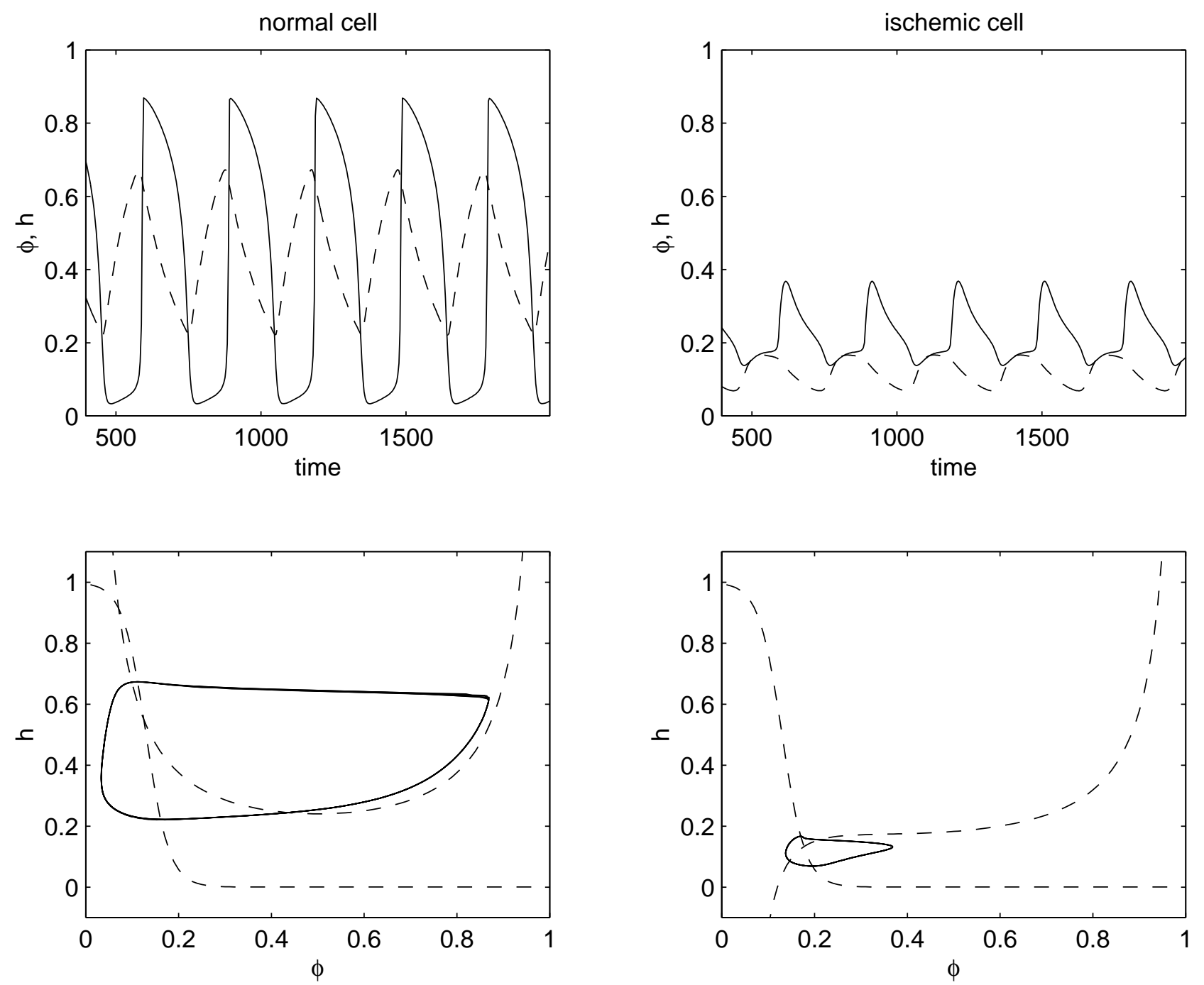

Figure 2: Oscillatory behavior of two coupled cells, one of which (left panels) has normal excitability and one of which (right panels) has an elevated rest potential $\left(\phi_{K}=0.12\right)$ and is inexcitable. The upper panels show the time course for the membrane potential and the inactivation variable $h$ for each cell, while the lower panels show trajectories in the $\phi-h$ phase plane. For this simulation $\frac{\delta}{\chi_{1}}=0.05, \frac{\delta}{\chi_{2}}=0.025$. 


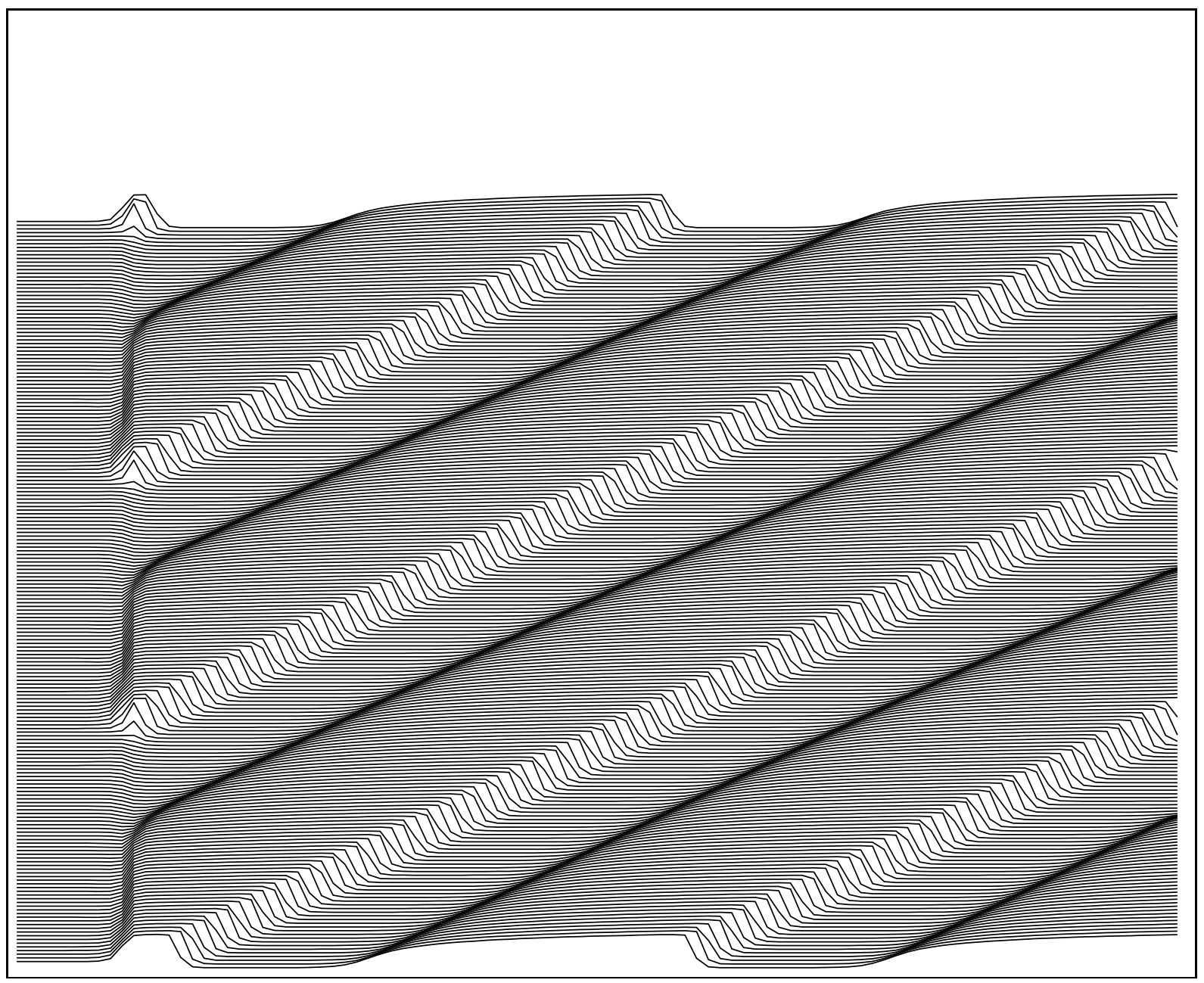

Figure 3: Periodic waves driven by an ischemic region with high extracellular potassium. For this simulation the cable length was $L=10$ and diffusion coefficient was $D=0.0001$. The ischemic region on the left has length $L=1$. Here the horizontal axis is space $x$ and the vertical axis is $\phi(x, t)+t$ (i.e., the potential $\phi(x, t)$ is shifted vertically by the amount $t$ ). 

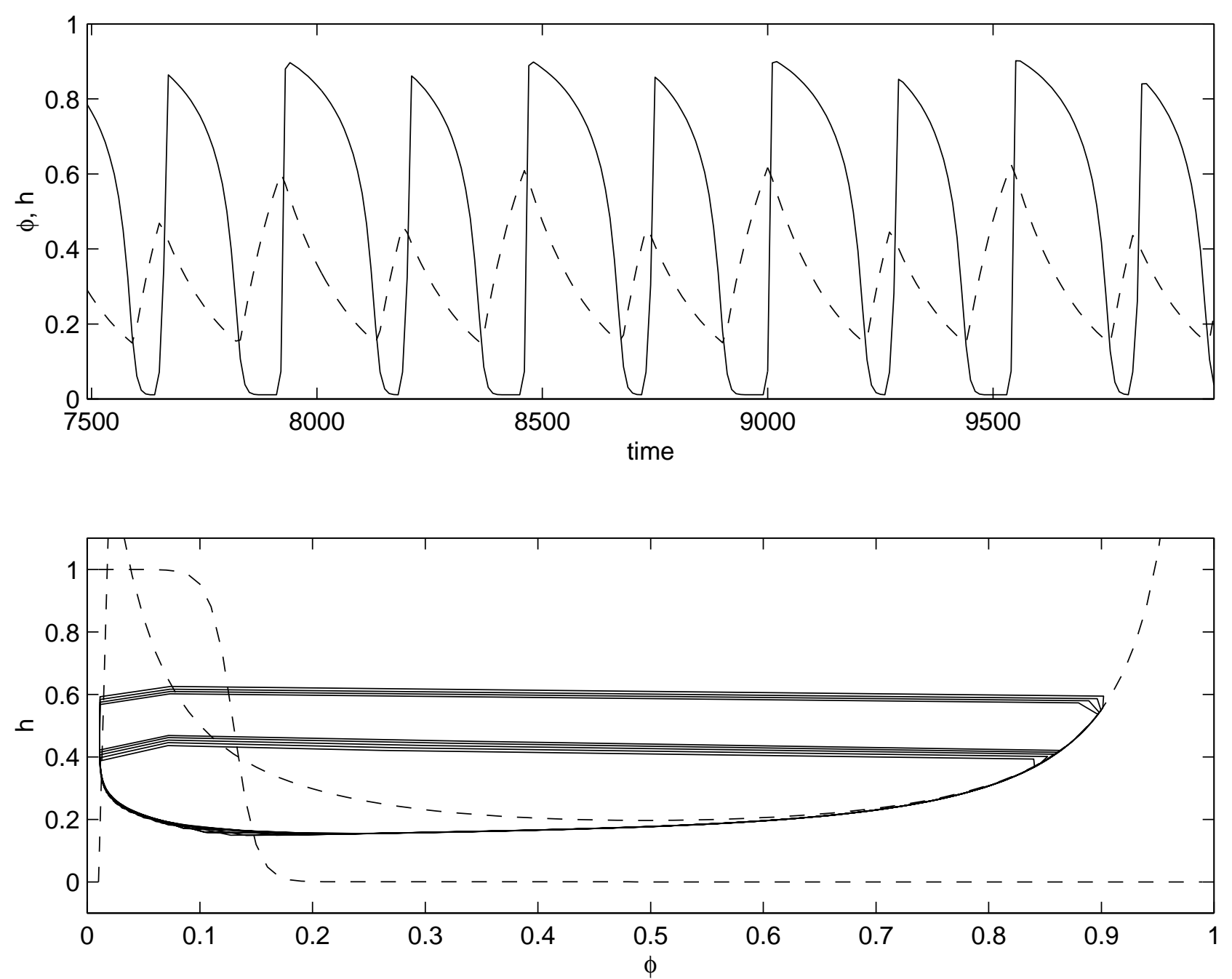

Figure 4: APD Alternans in a single cell in response to periodic stimuli with BCL of 270 ms. Upper panel: time course of the potential $\phi$ (solid curve) and sodium inactivation $h$ (dashed). Lower panel: trajectory in the $\phi-h$ phase plane. 

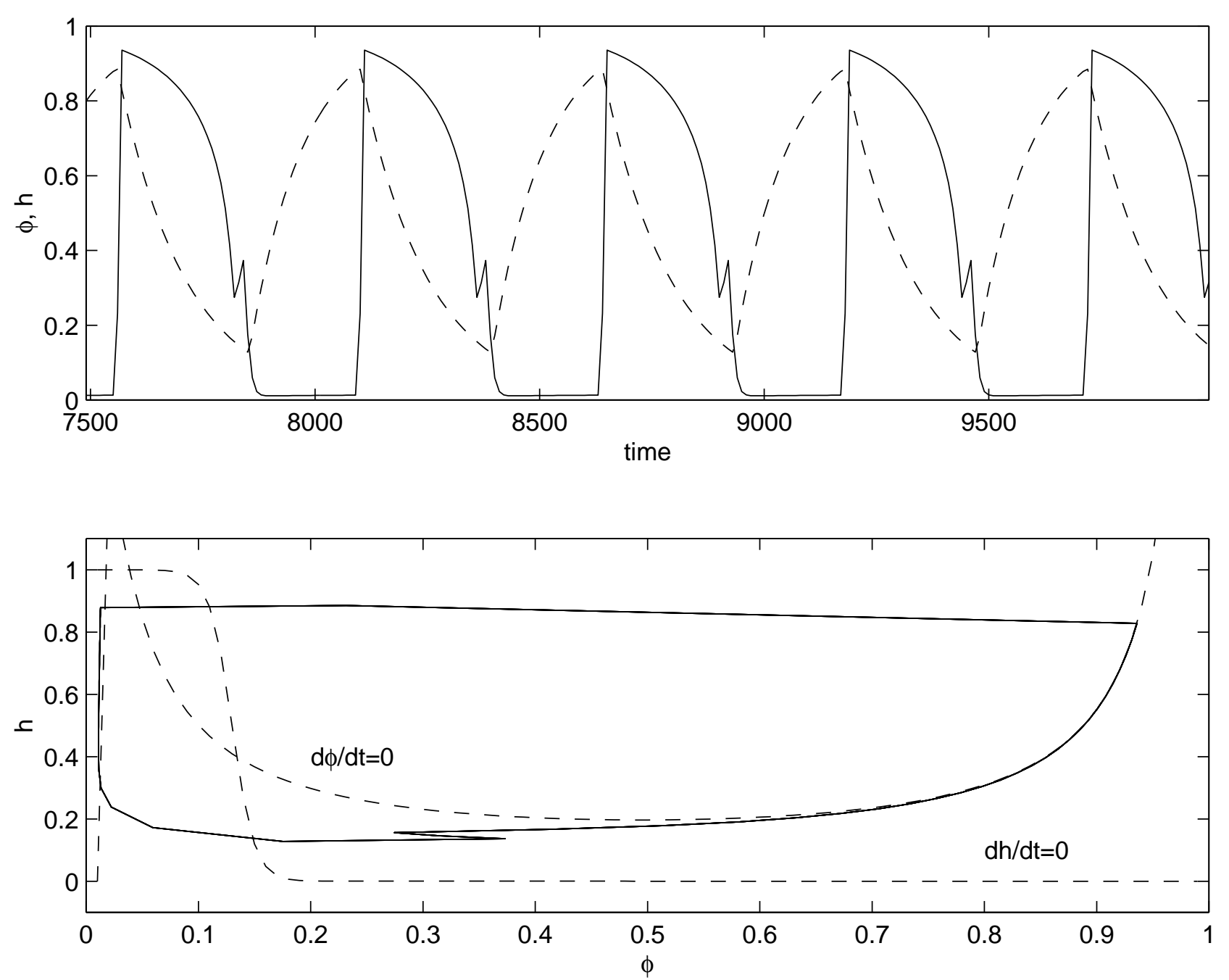

Figure 5: 2:1 action potential response to periodic stimuli with BCL of $270 \mathrm{~ms}$. Upper panel: time course of the potential $\phi$ (solid curve) and sodium inactivation $h$ (dashed). Lower panel: trajectory in the $\phi-h$ phase plane. 


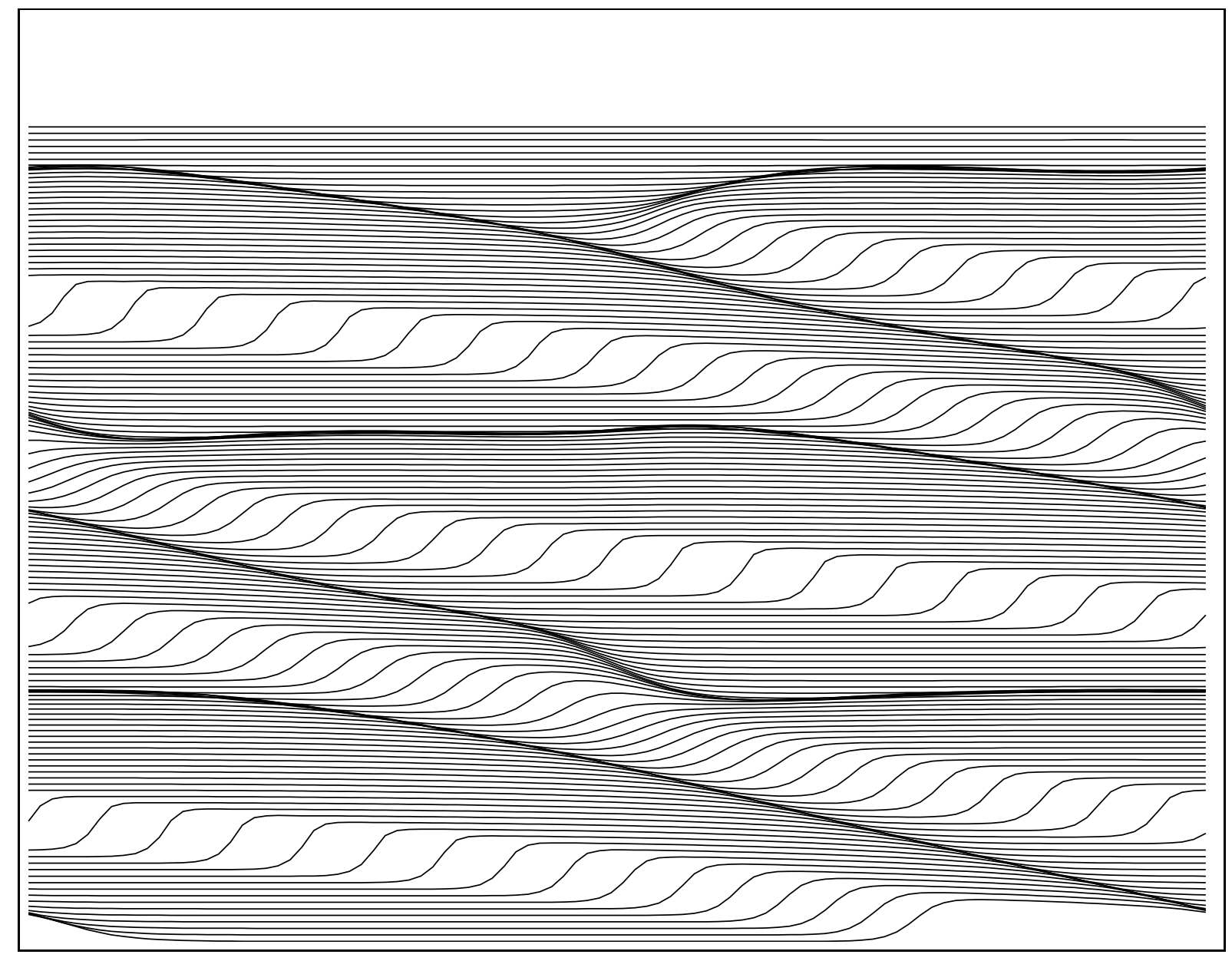

Figure 6: The breakup instability in one spatial dimension: Alternans on a ring leading to collapse. For this simulation the ring length was $L=1.3$ and diffusion coefficient was $D=0.0001$. Waves are initially propagating on a spatially homogeneous ring from right to left, and collapse after several rotations. As in Fig. 3, the horizontal axis is space $x$ and the vertical axis is $\phi(x, t)+t$. 


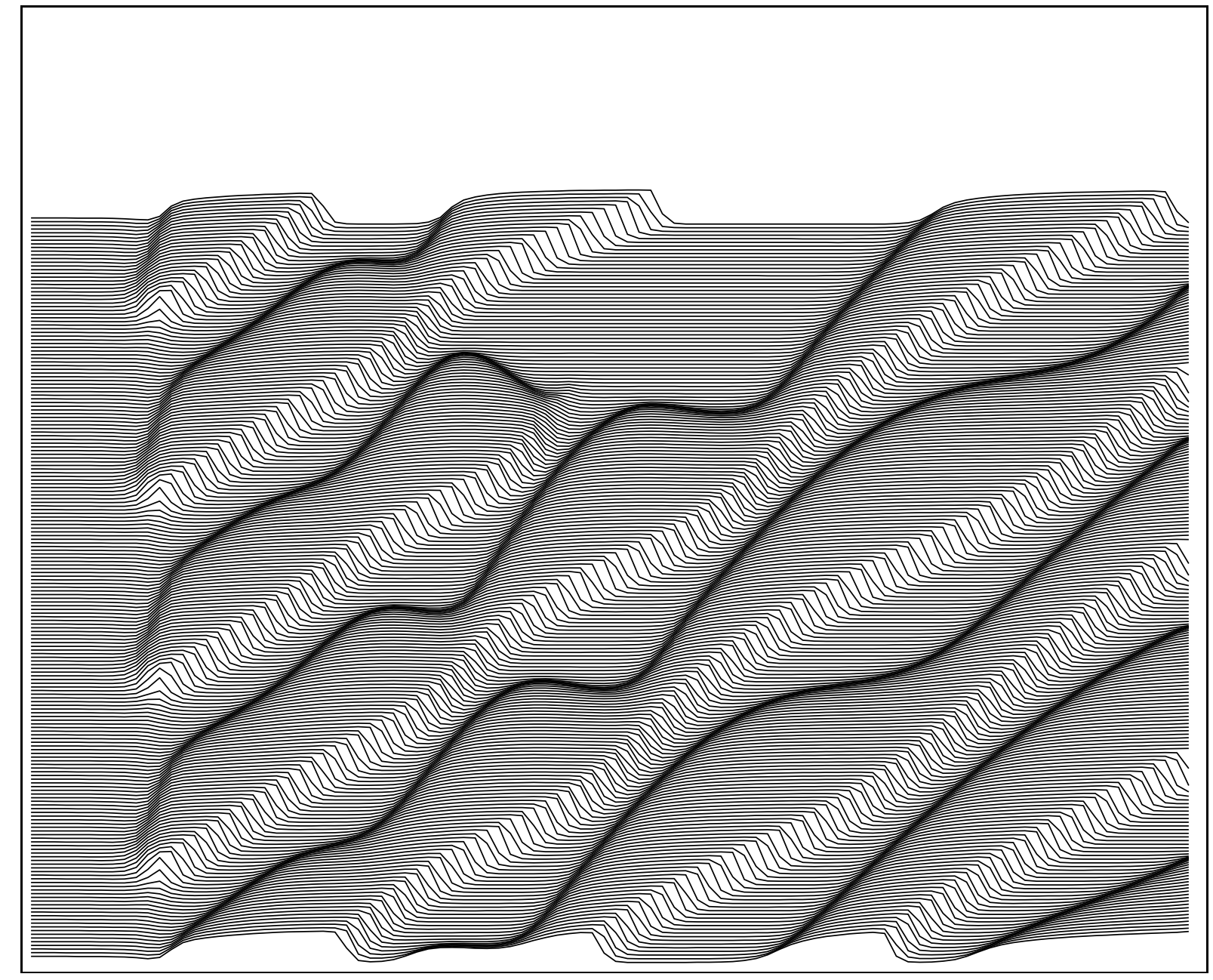

Figure 7: The breakup instability from a border zone: Recurrent alternans initiated from a border zone leading to propagation failure. The length of the cable is $L=10$ with diffusion coefficient $D=0.0001$. The ischemic zone on the left has width about $L=0.5$ and is separated from the normal region by a smooth transition of width about $L=1$. As in Fig. 3 the horizontal axis is space $x$ and the vertical axis is $\phi(x, t)+t$. 